\title{
Dirac Delta Function of Matrix Argument
}

\author{
Lin Zhang* \\ Institute of Mathematics, Hangzhou Dianzi University, Hangzhou 310018, PR China
}

\begin{abstract}
Dirac delta function of matrix argument is employed frequently in the development of diverse fields such as Random Matrix Theory, Quantum Information Theory, etc. The purpose of the article is pedagogical, it begins by recalling detailed knowledge about Heaviside unit step function and Dirac delta function. Then its extensions of Dirac delta function to vector spaces and matrix spaces are discussed systematically, respectively. The detailed and elementary proofs of these results are provided. Though we have not seen these results formulated in the literature, there certainly are predecessors. Applications are also mentioned.
\end{abstract}

\section{Heaviside unit step function $H$ and Dirac delta function $\delta$}

The materials in this section are essential from Hoskins' Book [4]. There are also no new results in this section. In order to be in a systematic way, it is reproduced here.

The Heaviside unit step function $H$ is defined as

$$
H(x):= \begin{cases}1, & x>0 \\ 0, & x<0\end{cases}
$$

That is, this function is equal to 1 over $(0,+\infty)$ and equal to 0 over $(-\infty, 0)$. This function can equally well have been defined in terms of a specific expression, for instance

$$
H(x)=\frac{1}{2}\left(1+\frac{x}{|x|}\right) \text {. }
$$

The value $H(0)$ is left undefined here. For all $x \neq 0$,

$$
H^{\prime}(x)=\frac{\mathrm{d} H(x)}{\mathrm{d} x}=0
$$

*E-mail: godyalin@163.com; linyz@zju.edu.cn 
corresponding to the obvious fact that the graph of the function $y=H(x)$ has zero slope for all $x \neq 0$. Naturally we describe the slope as "infinite" at origin. We shall denote by $\delta(x)$ the derivative of $H(x)$ :

$$
\delta(x)=H^{\prime}(x)=0, \quad \forall x \neq 0,
$$

and

$$
\delta(0)=+\infty
$$

We recall the definition of Dirac delta function:

Definition 1.1 (Dirac delta function). Dirac delta function $\delta(x)$ is defined by

$$
\delta(x)= \begin{cases}+\infty, & \text { if } x=0 \\ 0, & \text { if } x \neq 0\end{cases}
$$

Proposition 1.2 (Sampling property of the Dirac delta function). If $f$ is any function which is continuous on a neighborhood of 0 , then

$$
\int_{-\infty}^{+\infty} f(x) \delta(x) \mathrm{d} x=f(0)
$$

In fact, we have, for $a \neq 0$,

$$
\int_{-a}^{+a} f(x) \delta(x) \mathrm{d} x=f(0)
$$

and

$$
\int_{-\infty}^{a} \delta(x) \mathrm{d} x=\int_{-\infty}^{+\infty} H(a-x) \delta(x) \mathrm{d} x=H(a) .
$$

Definition 1.3. Assume that $f$ is continuous function which vanishes outside some finite interval. There corresponds a certain number which we write as $\langle H, f\rangle$, given by

$$
\langle H, f\rangle:=\int_{-\infty}^{+\infty} f(x) H(x) \mathrm{d} x=\int_{0}^{+\infty} f(x) \mathrm{d} x
$$

Similarly, $\langle\delta, f\rangle$ is given by

$$
\langle\delta, f\rangle:=\int_{-\infty}^{+\infty} f(x) \delta(x) \mathrm{d} x=f(0)
$$

For an ordinary function $f$ and a fixed $a \in \mathbb{R}$, the symbol $f_{a}$ denotes the translation of $f$ with respect to $a$ :

$$
f_{a}(x):=f(x-a)
$$

Thus

$$
\left\langle\delta_{a}, f\right\rangle=\int_{-\infty}^{+\infty} f(x) \delta_{a}(x) \mathrm{d} x=\int_{-\infty}^{+\infty} f(x) \delta(x-a) \mathrm{d} x=f(a)
$$


From the above discussion, it is easy to see that

$$
f(x) \delta(x-a)=f(x) \delta_{a}(x)=f(a) \delta(x)
$$

This fact will be used later.

Proposition 1.4. If $f$ is any function which has a continuous derivative $f^{\prime}$, at least in some neighborhood of 0 , then

$$
\left\langle\delta^{\prime}, f\right\rangle:=\int_{-\infty}^{+\infty} f(x) \delta^{\prime}(x) \mathrm{d} x=-\left\langle\delta, f^{\prime}\right\rangle=-f^{\prime}(0)
$$

Proof. Since

$$
\begin{aligned}
\int_{-\infty}^{+\infty} f(x) \frac{\delta(x)-\delta(x-\epsilon)}{\epsilon} \mathrm{d} x & =\frac{1}{\epsilon}\left(\int_{-\infty}^{+\infty} f(x) \delta(x) \mathrm{d} x-\int_{-\infty}^{+\infty} f(x) \delta(x-\epsilon) \mathrm{d} x\right) \\
& =\frac{f(0)-f(\epsilon)}{\epsilon}=-\frac{f(\epsilon)-f(0)}{\epsilon}
\end{aligned}
$$

it follows that

$$
\begin{aligned}
\left\langle\delta^{\prime}, f\right\rangle & =\int_{-\infty}^{+\infty} f(x) \delta^{\prime}(x) \mathrm{d} x=\int_{-\infty}^{+\infty} f(x) \lim _{\epsilon \rightarrow 0} \frac{\delta(x)-\delta(x-\epsilon)}{\epsilon} \mathrm{d} x \\
& =\lim _{\epsilon \rightarrow 0} \int_{-\infty}^{+\infty} f(x) \frac{\delta(x)-\delta(x-\epsilon)}{\epsilon} \mathrm{d} x \\
& =\lim _{\epsilon \rightarrow 0}-\frac{f(\epsilon)-f(0)}{\epsilon}=-f^{\prime}(0)=-\left\langle\delta, f^{\prime}\right\rangle .
\end{aligned}
$$

This completes the proof.

Proposition 1.5. The $n$-th derivative of the Dirac delta function, denoted by $\delta^{(n)}$, is defined by the following:

$$
\left\langle\delta^{(n)}, f\right\rangle=(-1)^{n} f^{(n)}(0)
$$

where $n \in \mathbb{N}_{+}$and $f$ is any function with continuous derivatives at least up to the $n$-th order in some neighborhood of 0 .

From these, we see that

$$
\begin{aligned}
\left\langle\delta_{a}^{\prime}, f\right\rangle & =-\left\langle\delta_{a}, f^{\prime}\right\rangle=-f^{\prime}(a) \\
\left\langle\delta_{a}^{(n)}, f\right\rangle & =(-1)^{n}\left\langle\delta_{a}, f^{(n)}\right\rangle=(-1)^{n} f^{(n)}(a) .
\end{aligned}
$$

Suppose that $g(t)$ increases monotonely over the closed interval $[a, b]$ : suppose there is $t_{0} \in(a, b)$ such that $g\left(t_{0}\right)=0$. We have known that

$$
\frac{\mathrm{d} g^{-1}(x)}{\mathrm{d} x}=\frac{1}{\frac{\mathrm{d} g(t)}{\mathrm{d} t}} .
$$


From this, we see that, via $x=g(t), t \in[a, b]$,

$$
\begin{aligned}
\int_{a}^{b} f(t) \delta(g(t)) \mathrm{d} t & =\int_{g(a)}^{g(b)} f\left(g^{-1}(x)\right) \delta(x) \mathrm{d}\left(g^{-1}(x)\right) \\
& =\int_{g(a)}^{g(b)} f\left(g^{-1}(x)\right) \delta(x) \frac{\mathrm{d} g^{-1}(x)}{\mathrm{d} x} \mathrm{~d} x \\
& =\left.f\left(g^{-1}(0)\right) \frac{\mathrm{d} g^{-1}(x)}{\mathrm{d} x}\right|_{x=g\left(t_{0}\right)}=\frac{f\left(t_{0}\right)}{g^{\prime}\left(t_{0}\right)}
\end{aligned}
$$

Proposition 1.6. If $g(t)$ is monotone, with $g(a)=0$ and $g^{\prime}(a) \neq 0$, then

$$
\delta(g(t))=\frac{\delta_{a}(t)}{\left|g^{\prime}(a)\right|}
$$

From this, we see that

$$
\delta(k x+b)=\frac{1}{|k|} \delta\left(x+\frac{b}{k}\right), \quad k \neq 0 .
$$

More generally, the delta distribution may be composed with a smooth function $g(x)$ in such a way that the familiar change of variables formula holds, that

$$
\int_{\mathbb{R}} f(g(x)) \delta(g(x))\left|g^{\prime}(x)\right| \mathrm{d} x=\int_{g(\mathbb{R})} f(t) \delta(t) \mathrm{d} t
$$

provided that $g$ is a continuously differentiable function with $g^{\prime}$ nowhere zero. That is, there is a unique way to assign meaning to the distribution $\delta \circ g$ so that this identity holds for all compactly supported test functions $f$. Therefore, the domain must be broken up to exclude the $g^{\prime}(x)=0$ point. This distribution satisfies $\delta(g(x))=0$ if $g$ is nowhere zero, and otherwise if $g$ has a real root at $x_{0}$, then

$$
\delta(g(x))=\frac{\delta\left(x-x_{0}\right)}{\left|g^{\prime}\left(x_{0}\right)\right|}
$$

It is natural to define the composition $\delta(g(x))$ for continuously differentiable functions $g$ by

$$
\delta(g(x))=\sum_{j} \frac{\delta\left(x-x_{j}\right)}{\left|g^{\prime}\left(x_{j}\right)\right|}
$$

where the sum extends over all roots of $g(x)$, which are assumed to be simple. Thus, for example

$$
\delta\left(x^{2}-a^{2}\right)=\frac{1}{2|a|}(\delta(x+a)+\delta(x-a)) .
$$

In the integral form the generalized scaling property may be written as

$$
\int_{-\infty}^{+\infty} f(x) \delta(g(x)) \mathrm{d} x=\sum_{j} \frac{f\left(x_{j}\right)}{\left|g^{\prime}\left(x_{j}\right)\right|} .
$$


Example 1.7 ([1]). If $T$ is an $n \times n$ positive definite matrix and $r \in \mathbb{R}^{+}, \alpha \geqslant 0$, then

$$
\int_{B_{n}(T, r)}(r-\langle\boldsymbol{u}|T| \boldsymbol{u}\rangle)^{\alpha}[\mathrm{d} \boldsymbol{u}]=\frac{\pi^{n} \Gamma(\alpha+1)}{\Gamma(n+\alpha+1)} \frac{r^{n+\alpha}}{\operatorname{det}(T)},
$$

where $B_{n}(T, r):=\left\{\boldsymbol{u} \in \mathbb{C}^{n} \mid\langle\boldsymbol{u}|T| \boldsymbol{u}\rangle<r\right\}$ and $[\mathrm{d} \boldsymbol{u}]=\prod_{j=1}^{n} \mathrm{~d} u_{j}$ for $[\mathrm{d} z]=\mathrm{d}(\operatorname{Re}(z)) \mathrm{d}(\operatorname{Im}(z))$.

Indeed, let

$$
\boldsymbol{v}=r^{-\frac{1}{2}} T^{\frac{1}{2}} \boldsymbol{u}
$$

Then $[\mathrm{d} v]=\operatorname{det}\left(r^{-1} T\right)[\mathrm{d} \boldsymbol{u}]$ or $[\mathrm{d} \boldsymbol{u}]=\operatorname{det}\left(r T^{-1}\right)[\mathrm{d} \boldsymbol{v}]$. Thus

$$
\int_{B_{n}(T, r)}(r-\langle\boldsymbol{u}|T| \boldsymbol{u}\rangle)^{\alpha}[\mathrm{d} \boldsymbol{u}]=\frac{r^{n+\alpha}}{\operatorname{det}(T)} \int_{B_{n}\left(\mathbb{1}_{n}, 1\right)}(1-\langle\boldsymbol{v} \mid \boldsymbol{v}\rangle)^{\alpha}[\mathrm{d} \boldsymbol{v}],
$$

where $B_{n}\left(\mathbb{1}_{n}, 1\right)=\left\{\boldsymbol{v} \in \mathbb{C}^{n} \mid\langle\boldsymbol{v} \mid \boldsymbol{v}\rangle<1\right\}$. Now

$$
B_{n}\left(\mathbb{1}_{n}, 1\right)=\cup_{\gamma \in[0,1)} S_{n}(\gamma),
$$

where $S_{n}(\gamma)=\left\{v \in \mathbb{C}^{n} \mid\|v\|_{2}=\gamma\right\}$.

$$
\begin{aligned}
\int_{B_{n}\left(\mathbb{1}_{n}, 1\right)}(1-\langle\boldsymbol{v} \mid \boldsymbol{v}\rangle)^{\alpha}[\mathrm{d} \boldsymbol{v}] & =\int_{0}^{1} \mathrm{~d} \gamma \int \delta\left(\gamma-\|\boldsymbol{v}\|_{2}\right)(1-\langle\boldsymbol{v} \mid \boldsymbol{v}\rangle)^{\alpha}[\mathrm{d} \boldsymbol{v}] \\
& =\int_{0}^{1} \mathrm{~d} \gamma\left(1-\gamma^{2}\right)^{\alpha} \cdot \int \delta\left(\gamma-\|\boldsymbol{v}\|_{2}\right)[\mathrm{d} \boldsymbol{v}] \\
& =\int_{0}^{1} \mathrm{~d} \gamma\left(1-\gamma^{2}\right)^{\alpha} \operatorname{vol}\left(S_{n}(\gamma)\right)
\end{aligned}
$$

where

$$
\operatorname{vol}\left(S_{n}(\gamma)\right)=\int \delta\left(\gamma-\|v\|_{2}\right)[\mathrm{d} v]=\frac{2 \pi^{n}}{\Gamma(n)} \gamma^{2 n-1}
$$

Therefore, we obtain that

$$
\begin{aligned}
\int_{B_{n}(T, r)}(r-\langle\boldsymbol{u}|T| \boldsymbol{u}\rangle)^{\alpha}[\mathrm{d} \boldsymbol{u}] & =\frac{2 \pi^{n}}{\Gamma(n)} \frac{r^{n+\alpha}}{\operatorname{det}(T)} \int_{0}^{1}\left(1-\gamma^{2}\right)^{\alpha} \gamma^{2 n-1} \mathrm{~d} \gamma \\
& =\frac{\pi^{n}}{\Gamma(n)} \frac{r^{n+\alpha}}{\operatorname{det}(T)} \int_{0}^{1}\left(1-\gamma^{2}\right)^{\alpha} \gamma^{2 n-2} \mathrm{~d}\left(\gamma^{2}\right)
\end{aligned}
$$

where

$$
\int_{0}^{1}\left(1-\gamma^{2}\right)^{\alpha} \gamma^{2 n-2} \mathrm{~d}\left(\gamma^{2}\right)=\int_{0}^{1}(1-x)^{\alpha} x^{n-1} \mathrm{~d} x=B(\alpha+1, n)=\frac{\Gamma(\alpha+1) \Gamma(n)}{\Gamma(n+\alpha+1)} .
$$

This completes the proof.

Definition 1.8 (Convolution). An operation on functions, called convolution and denoted by the $\operatorname{symbol} *$, is defined by:

$$
f * g(x)=\int_{\mathbb{R}} f(x-t) g(t) \mathrm{d} t=\int_{\mathbb{R}} f(t) g(x-t) \mathrm{d} t
$$


It is easily seen that some properties of convolution:

(i) $\delta_{a} * \delta_{b}=\delta_{a+b}$.

(ii) The delta function as a convolution unit: $\delta * f=f * \delta=f$.

(iii) Convolution as the translation: $\delta_{a} * f=f * \delta_{a}=f_{a}$, where $f_{a}(x):=f(x-a)$.

(iv) $\delta^{(n)} * f=f * \delta^{(n)}=f^{(n)}$.

Definition 1.9 (Fourier transform). Let $f$ be a complex-valued function of the real variable $t$ which is absolutely integrable over the whole real axis $\mathbb{R}$. That is,

$$
\int_{\mathbb{R}}\left|f_{1}(x)\right|<+\infty \text { and } \int_{\mathbb{R}}\left|f_{2}(x)\right|<+\infty,
$$

where $f=f_{1}+\sqrt{-1} f_{2}$. We define the Fourier transform of $f$ to be a new function

$$
\widehat{f}(\omega):=\mathcal{F}(f)(\omega)=\int_{\mathbb{R}} e^{-\mathrm{i} \omega t} f(t) \mathrm{d} t
$$

Next, we consider the Fourier integral representation of Dirac delta function which is very powerful in applications. We use the following standard result:

$$
\mathrm{PV} \int_{-\infty}^{+\infty} \frac{e^{\mathrm{i} \omega x}}{x} \mathrm{~d} x=\mathrm{i} \pi
$$

where the symbol PV denotes the Cauchy Principal Value of the integral and $\omega>0$ a constant. That is,

$$
\int_{-\infty}^{+\infty} \frac{\sin (\omega x)}{x} \mathrm{~d} x=\pi \text { and } \int_{-\infty}^{+\infty} \frac{\cos (\omega x)}{x} \mathrm{~d} x=0
$$

Replacing $\omega$ by $-\omega$ simply changes the sign of the first of these two real integrals and leaves the other unaltered. That is, if $\omega>0$

$$
\mathrm{PV} \int_{-\infty}^{+\infty} \frac{e^{-\mathrm{i} \omega x}}{x} \mathrm{~d} x=-\mathrm{i} \pi
$$

Hence, if we replace $\omega$ by the usual symbol $t$ for the independent real variable we can write

$$
\mathrm{PV} \int_{-\infty}^{+\infty} \frac{e^{\mathrm{i} t x}}{x} \mathrm{~d} x=\mathrm{i} \pi \operatorname{sign}(t)
$$

i.e.,

$$
\frac{1}{2 \pi} \int_{-\infty}^{+\infty} \frac{e^{\mathrm{i} t x}}{\mathrm{i} x} \mathrm{~d} x=\frac{1}{2} \operatorname{sign}(t)= \begin{cases}\frac{1}{2}, & t>0 \\ -\frac{1}{2}, & t<0\end{cases}
$$

A formal differentiation of this with respect to $t$ then yields the following result: 
Proposition 1.10. It holds that

$$
\delta(t)=\frac{1}{2 \pi} \int_{-\infty}^{+\infty} e^{\mathrm{i} t x} \mathrm{~d} x .
$$

This amounts to say $\mathcal{F}^{-1}(1)(t)=\delta(t)$. Replacing $t$ by $t-a$, we have

$$
\frac{1}{2 \pi} \int_{-\infty}^{+\infty} e^{\mathrm{i}(t-a) x} \mathrm{~d} x=\delta(t-a)=\delta_{a}(t)
$$

This amounts to say $\mathcal{F}^{-1}\left(e^{-\mathrm{i} a x}\right)(t)=\delta_{a}(t)$ or $\mathcal{F}\left(\delta_{a}(t)\right)(x)=e^{-\mathrm{i} a x}$. The integral on the lefthand side of (1.40) is, of course, divergent, and it is clear that this equation must be understood symbolically. That is to say, for all sufficiently well-behaved functions $f$, we should interpret (1.40) to mean that

$$
\int_{-\infty}^{+\infty} f(t)\left[\frac{1}{2 \pi} \int_{-\infty}^{+\infty} e^{\mathrm{i} t \omega} \mathrm{d} \omega\right] \mathrm{d} t=\int_{-\infty}^{+\infty} f(t) \delta(t) \mathrm{d} t=f(0)
$$

or, more generally, that

$$
\int_{-\infty}^{+\infty} f(x)\left[\frac{1}{2 \pi} \int_{-\infty}^{+\infty} e^{\mathrm{i}(t-x) \omega} \mathrm{d} \omega\right] \mathrm{d} x=\int_{-\infty}^{+\infty} f(x) \delta(t-x) \mathrm{d} x=f(t) .
$$

We can rewrite this result in the form

$$
\begin{aligned}
f(t) & =\int_{-\infty}^{+\infty} f(x)\left[\frac{1}{2 \pi} \int_{-\infty}^{+\infty} e^{\mathrm{i}(t-x) \omega} \mathrm{d} \omega\right] \mathrm{d} x \\
& =\frac{1}{2 \pi} \int_{-\infty}^{+\infty} e^{\mathrm{i} t \omega}\left[\int_{-\infty}^{+\infty} f(x) e^{-\mathrm{i} x \omega} \mathrm{d} x\right] \mathrm{d} \omega=\frac{1}{2 \pi} \int_{-\infty}^{+\infty} e^{\mathrm{i} t \omega} \widehat{f}(\omega) \mathrm{d} \omega
\end{aligned}
$$

Proposition 1.11 (Fourier Inversion). Let $f$ be a (real or complex valued) function of a single real variable which is absolutely integrable over the interval $(-\infty,+\infty)$ and which also satisfies the Dirichlet conditions over every finite interval. If $\widehat{f}(\omega)$ denotes the Fourier transform of $f$, then at each point $t$ we have

$$
\frac{1}{2 \pi} \int_{-\infty}^{+\infty} e^{\mathrm{i} t \omega} \widehat{f}(\omega) \mathrm{d} \omega=\frac{1}{2}[f(t+)+f(t-)],
$$

where $f(t \pm):=\lim _{s \rightarrow t^{ \pm}} f(s)$.

There are several important properties of the Fourier transform which merit explicit mention.

(i) The Fourier transform of the convolution of two functions is equal to the product of their individual transforms: $\mathcal{F}(f * g)=\mathcal{F}(f) \mathcal{F}(g)$.

(ii) $\mathcal{F}(f g)=\frac{1}{2 \pi} \mathcal{F}(f) * \mathcal{F}(g)$.

(iii) The Fourier transformation is linear: $\mathcal{F}\left(\lambda_{1} f_{1}+\lambda_{2} f_{2}\right)=\lambda_{1} \mathcal{F}\left(f_{1}\right)+\lambda_{2} \mathcal{F}\left(f_{2}\right)$. 
(iv) $\mathcal{F}(f(x-a))(\omega)=e^{-i \omega a} \mathcal{F}(f(x))(\omega)$.

(v) $\mathcal{F}\left(f(x) e^{-a x}\right)(\omega)=\mathcal{F}(f(x))(a+\mathrm{i} \omega)$.

(vi) $\mathcal{F}\left(f^{\prime}\right)(\omega)=\mathrm{i} \omega \mathcal{F}(f)(\omega)$.

(vii) $\mathcal{F}(f(a x))(\omega)=\frac{1}{a} \mathcal{F}(f(x))\left(\frac{\omega}{a}\right)$.

For example, the proofs of (iv) and (vi) are given. Indeed, $f(x-a)=f_{a}(x)=f * \delta_{a}(x)$, thus $\mathcal{F}(f(x-a))(\omega)=\mathcal{F}\left(f * \delta_{a}(x)\right)(\omega)=\mathcal{F}(f) \mathcal{F}\left(\delta_{a}\right)(\omega)$, that is, $\mathcal{F}(f(x-a))(\omega)=e^{-i \omega a} \mathcal{F}(f)$, hence (iv). Since $f^{\prime}=f * \delta^{\prime}$, it follows that $\mathcal{F}\left(f^{\prime}\right)=\mathcal{F}\left(f * \delta^{\prime}\right)=\mathcal{F}(f) \mathcal{F}\left(\delta^{\prime}\right)$. In what follows, we calculate $\mathcal{F}\left(\delta^{\prime}\right)$. By definition of Fourier transform,

$$
\mathcal{F}\left(\delta^{\prime}\right)(\omega)=\int_{\mathbb{R}} e^{-\mathrm{i} \omega t} \delta^{\prime}(t) \mathrm{d} t=-\left.\frac{\mathrm{d} e^{-\mathrm{i} \omega t}}{\mathrm{~d} t}\right|_{t=0}=\mathrm{i} \omega
$$

Thus $\mathcal{F}\left(f^{\prime}\right)(\omega)=\mathrm{i} \omega \mathcal{F}(f)(\omega)$, hence (vi). This property can be generalized: $\mathcal{F}\left(f^{(n)}\right)(\omega)=$ $(\mathrm{i} \omega)^{n} \mathcal{F}(f)(\omega)$. Indeed,

$$
\mathcal{F}\left(f^{(n)}\right)(\omega)=\mathcal{F}\left(f * \delta^{(n)}\right)(\omega)=\mathcal{F}(f)(\omega) \mathcal{F}\left(\delta^{(n)}\right)(\omega)=(\mathrm{i} \omega)^{n} \mathcal{F}(f)(\omega) .
$$

We can apply the sampling property of the delta function to the Fourier inversion integral:

$$
\frac{1}{2 \pi} \int_{-\infty}^{+\infty} e^{\mathrm{i} x \omega} \delta(\omega-\alpha) \mathrm{d} \omega=\frac{1}{2 \pi} e^{\mathrm{i} x \alpha}
$$

and similarly

$$
\frac{1}{2 \pi} \int_{-\infty}^{+\infty} e^{\mathrm{i} x \omega} \delta(\omega+\alpha) \mathrm{d} \omega=\frac{1}{2 \pi} e^{-\mathrm{i} x \alpha}
$$

Thus, recalling that the Fourier transform is defined in general for complex-valued functions, these results suggest that we can give the following definitions for the Fourier transforms of complex exponentials such as

$$
\mathcal{F}\left(e^{\mathrm{i} \alpha x}\right)(\omega)=2 \pi \delta(\omega-\alpha) ; \quad \mathcal{F}\left(e^{-\mathrm{i} \alpha x}\right)(\omega)=2 \pi \delta(\omega+\alpha)
$$

Both equations immediately yield the following definitions for the Fourier transforms of the real functions $\cos (\alpha x)$ and $\sin (\alpha x)$ :

$$
\begin{gathered}
\mathcal{F}(\cos (\alpha x))(\omega)=\pi(\delta(\omega-\alpha)+\delta(\omega+\alpha)), \\
\mathcal{F}(\sin (\alpha x))(\omega)=-\mathrm{i} \pi(\delta(\omega-\alpha)-\delta(\omega+\alpha)) .
\end{gathered}
$$

In particular, taking $\alpha=0$, we find that the generalized Fourier transform of the constant function $f(t) \equiv 1$ is simply $2 \pi \delta(\omega)$. This in turn allows us to offer a definition of the Fourier transform of the unit step function. 
Proposition 1.12. The Fourier transform of the Heaviside step function $H(x)=\frac{1}{2}+\frac{1}{2} \operatorname{sign}(x)$, where $\operatorname{sign}(x)=\frac{x}{|x|}$, is given by

$$
\widehat{H}(\omega)=\pi \delta(\omega)+\frac{1}{\mathrm{i} \omega}
$$

Proof. Now

$$
\frac{1}{2 \pi} \int_{-\infty}^{+\infty} \frac{e^{\mathrm{i} x \omega}}{\mathrm{i} \omega} \mathrm{d} \omega=\frac{1}{2} \operatorname{sign}(x)= \begin{cases}\frac{1}{2}, & x>0 \\ -\frac{1}{2}, & x<0\end{cases}
$$

Then we know that $\frac{2}{\mathrm{i} \omega}$ is a suitable choice for the Fourier transform of the function $\operatorname{sign}(x)$ in the sense that

$$
\operatorname{sign}(x)=\frac{1}{2 \pi} \int_{-\infty}^{+\infty} e^{\mathrm{i} x \omega} \frac{2}{\mathrm{i} \omega} \mathrm{d} \omega=\mathcal{F}^{-1}\left(\frac{2}{\mathrm{i} \omega}\right)(x) .
$$

This amounts to say that $\mathcal{F}(\operatorname{sign}(x))(\omega)=\frac{2}{\mathrm{i} \omega}$. Hence for Heaviside step function

$$
H(x)=\frac{1}{2}+\frac{1}{2} \operatorname{sign}(x),
$$

the Fourier transform of it is given by

$$
\mathcal{F}(H(x))(\omega)=\mathcal{F}\left(\frac{1}{2}+\frac{1}{2} \operatorname{sign}(x)\right)=\frac{1}{2} \mathcal{F}(1)(\omega)+\frac{1}{2} \mathcal{F}(\operatorname{sign}(x))(\omega),
$$

i.e.

$$
\mathcal{F}(H(x))(\omega)=\pi \delta(\omega)+\frac{1}{\mathrm{i} \omega} \Longleftrightarrow \widehat{H}(\omega)=\pi \delta(\omega)+\frac{1}{\mathrm{i} \omega} .
$$

This completes the proof.

We can get some important properties of Dirac delta function which are listed below:

(i) The delta function is an even distribution: $\delta(x)=\delta(-x)$.

(ii) The delta function satisfies the following scaling property for a non-zero scalar: $\delta(a x)=$ $\frac{1}{|a|} \delta(x)$ for $a \in \mathbb{R} \backslash\{0\}$.

(iii) The distributional product of $\delta(x)$ and $x$ is equal to zero: $x \delta(x)=0$.

(iv) If $x f(x)=x g(x)$, where $f$ and $g$ are distributions, then $f(x)=g(x)+c \delta(x)$ for some constant $c$. 
Previous two facts can be checked as follows: Note that

$$
\begin{aligned}
\delta(-x) & =\frac{1}{2 \pi} \int_{-\infty}^{\infty} e^{-\mathrm{i} t x} \mathrm{~d} t=-\frac{1}{2 \pi} \int_{-\infty}^{\infty} e^{-\mathrm{i} t x} \mathrm{~d}(-t) \\
& =-\frac{1}{2 \pi} \int_{+\infty}^{-\infty} e^{\mathrm{i} s x} \mathrm{~d} s=\frac{1}{2 \pi} \int_{-\infty}^{\infty} e^{\mathrm{i} s x} \mathrm{~d} s=\delta(x) .
\end{aligned}
$$

This is (i). For the proof of (ii), since $a \neq 0$, we observe that $\delta(a x)=\delta(-a x)$ by (i), hence $\delta(a x)=\delta(|a| x)$, it follows that

$$
\delta(a x)=\delta(|a| x)=\frac{1}{2 \pi} \int_{-\infty}^{+\infty} e^{\mathrm{i} t \cdot|a| x} \mathrm{~d} t=\frac{1}{2 \pi} \int_{-\infty}^{+\infty} e^{\mathrm{i}|a| t \cdot x} \mathrm{~d} t .
$$

Let $s=|a| t$. Then $\mathrm{d} s=|a| \mathrm{d} t$, thus

$$
\delta(a x)=\frac{1}{|a|} \frac{1}{2 \pi} \int_{-\infty}^{+\infty} e^{\mathrm{i} s \cdot x} \mathrm{~d} s=\frac{1}{|a|} \delta(x) .
$$

That is, in the sense of distribution,

$$
\delta(a x)=\frac{1}{|a|} \delta(x), \quad a \in \mathbb{R} \backslash\{0\} .
$$

\section{Dirac delta function of vector argument}

Definition 2.1 (Dirac delta function of real-vector arguments). The real-vector delta function can be defined in $n$-dimensional Euclidean space $\mathbb{R}^{n}$ as the measure such that

$$
\int_{\mathbb{R}^{n}} f(\boldsymbol{x}) \delta(\boldsymbol{x})[\mathrm{d} \boldsymbol{x}]=f(\mathbf{0})
$$

for every compactly supported continuous function $f$. As a measure, the $n$-dimensional delta function is the product measure of the 1-dimensional delta functions in each variable separately. Thus, formally, with

$$
\delta(x)=\prod_{j=1}^{n} \delta\left(x_{j}\right)
$$

where $x=\left[x_{1}, \ldots, x_{n}\right]^{\top} \in \mathbb{R}^{n}$.

The delta function in an $n$-dimensional space satisfies the following scaling property instead:

$$
\delta(a x)=|a|^{-n} \delta(x), \quad a \in \mathbb{R} \backslash\{0\}
$$

Indeed, $a x=\left[a x_{1}, \ldots, a x_{n}\right]^{\top}$ for $x=\left[x_{1}, \ldots, x_{n}\right]^{\top}$, thus

$$
\delta(a x)=\prod_{j=1}^{n} \delta\left(a x_{j}\right)=\prod_{j=1}^{n}|a|^{-1} \delta\left(x_{j}\right)=|a|^{-n} \prod_{j=1}^{n} \delta\left(x_{j}\right)=|a|^{-n} \delta(x) .
$$


This indicates that $\delta$ is a homogeneous distribtion of degree $(-n)$. As in the one-variable case, it is possible to define the compositon of $\delta$ with a bi-Lipschitz function $g: \mathbb{R}^{n} \rightarrow \mathbb{R}^{n}$ uniquely so that the identity

$$
\int_{\mathbb{R}^{n}} f(g(\boldsymbol{x})) \delta(g(\boldsymbol{x}))\left|\operatorname{det} g^{\prime}(\boldsymbol{x})\right|[\mathrm{d} \boldsymbol{x}]=\int_{g\left(\mathbb{R}^{n}\right)} f(\boldsymbol{u}) \delta(\boldsymbol{u})[\mathrm{d} \boldsymbol{u}]
$$

for all compactly supported functions $f$.

Using the coarea formula from geometric measure theory, one can also define the composition of the delta function with a submersion from one Euclidean space to another one of different dimension; the result is a type of current. In the special case of a continuously differentiable function $g: \mathbb{R}^{n} \rightarrow \mathbb{R}$ such that the gradient of $g$ is nowhere zero, the following identity holds ${ }^{1}$

$$
\int_{\mathbb{R}^{n}} f(\boldsymbol{x}) \delta(g(\boldsymbol{x}))[\mathrm{d} \boldsymbol{x}]=\int_{g^{-1}(0)} \frac{f(\boldsymbol{x})}{|\nabla g(\boldsymbol{x})|} \mathrm{d} \sigma(\boldsymbol{x})
$$

where the integral on the right is over $g^{-1}(0)$, the $(n-1)$-dimensional surface defined by $g(x)=0$ with respect to the Minkowski content measure. That is known as a simple layer integral.

Proposition 2.2. It holds that

$$
\delta(x)=\frac{1}{(2 \pi)^{n}} \int_{\mathbb{R}^{n}} e^{\mathrm{i}\langle t, x\rangle}[\mathrm{d} \boldsymbol{t}] \quad\left(\boldsymbol{x} \in \mathbb{R}^{n}\right) .
$$

Proof. Let $x=\left[x_{1}, \ldots, x_{n}\right]^{\top} \in \mathbb{R}^{n}$. Then by Fourier transform of Dirac delta function:

$$
\begin{aligned}
\delta(x) & =\prod_{j=1}^{n} \delta\left(x_{j}\right)=\prod_{j=1}^{n} \frac{1}{2 \pi} \int_{\mathbb{R}} e^{\mathrm{i} t_{j} x_{j}} \mathrm{~d} t_{j} \\
& =\frac{1}{(2 \pi)^{n}} \int_{\mathbb{R}^{n}} e^{\mathrm{i} \sum_{j=1}^{n} t_{j} x_{j}} \prod_{j=1}^{n} \mathrm{~d} t_{j} \\
& =\frac{1}{(2 \pi)^{n}} \int_{\mathbb{R}^{n}} e^{\mathrm{i}\langle\boldsymbol{t}, \boldsymbol{x}\rangle}[\mathrm{d} \boldsymbol{t}] \quad\left(\boldsymbol{x} \in \mathbb{R}^{n}\right),
\end{aligned}
$$

where $[\mathrm{d} t]:=\prod_{j=1}^{n} \mathrm{~d} t_{j}$

Proposition 2.3. For a full-ranked real matrix $A \in \mathbb{R}^{n \times n}$, it holds that

$$
\delta(A x)=\frac{1}{|\operatorname{det}(A)|} \delta(x), \quad x \in \mathbb{R}^{n}
$$

In particular, under any reflection or rotation $R$, the delta function is invariant:

$$
\delta(R x)=\delta(x) .
$$

\footnotetext{
${ }^{1}$ See https://en.wikipedia.org/wiki/Dirac_delta_function
} 
The first proof. By using Fourier transform of Dirac delta function, it follows that

$$
\delta(A \boldsymbol{x})=\frac{1}{(2 \pi)^{n}} \int_{\mathbb{R}^{n}} e^{\mathrm{i}\langle\boldsymbol{t}, A x\rangle}[\mathrm{d} \boldsymbol{t}]=\frac{1}{(2 \pi)^{n}} \int_{\mathbb{R}^{n}} e^{\mathrm{i}\left\langle A^{\top} \boldsymbol{t}, \boldsymbol{x}\right\rangle}[\mathrm{d} \boldsymbol{t}] \quad\left(\boldsymbol{x} \in \mathbb{R}^{n}\right) .
$$

Let $s=A^{\top} \boldsymbol{t}$. Then $[\mathrm{d} s]=\left|\operatorname{det}\left(A^{\top}\right)\right|[\mathrm{d} \boldsymbol{t}]=|\operatorname{det}(A)|[\mathrm{d} \boldsymbol{t}]$. From this, we see that

$$
\delta(A \mathbf{x})=\left|\operatorname{det}^{-1}(A)\right| \frac{1}{(2 \pi)^{n}} \int_{\mathbb{R}^{n}} e^{\mathrm{i}\langle\boldsymbol{s}, \boldsymbol{x}\rangle}[\mathrm{d} \boldsymbol{s}]=\left|\operatorname{det}^{-1}(A)\right| \delta(\boldsymbol{x}) \quad\left(\boldsymbol{x} \in \mathbb{R}^{n}\right) .
$$

Thus $\delta(R x)=\delta(x)$ since $\operatorname{det}(R)= \pm 1$ for reflection or any rotation $R$. We are done.

The second proof. By SVD, we have two orthogonal matrices $L, R$ and diagonal matrix

$$
\Lambda=\operatorname{diag}\left(\lambda_{1}, \ldots, \lambda_{n}\right)
$$

with positive diagonal entries such that $A=L \Lambda R^{\top}$. Then, via $y:=R^{\top} x$ (hence $\delta(y)=\delta(x)$ ),

$$
\delta(A \boldsymbol{x})=\delta\left(L \Lambda R^{\top} \boldsymbol{x}\right)=\delta(\Lambda \boldsymbol{y})=\prod_{j=1}^{n} \delta\left(\lambda_{j} y_{j}\right)=\prod_{j=1}^{n} \lambda_{j}^{-1} \delta\left(y_{j}\right),
$$

that is,

$$
\delta(A \boldsymbol{x})=\frac{1}{\prod_{j=1}^{n} \lambda_{j}} \prod_{j=1}^{n} \delta\left(y_{j}\right)=\frac{1}{\prod_{j=1}^{n} \lambda_{j}} \delta(\boldsymbol{y})=\frac{1}{\prod_{j=1}^{n} \lambda_{j}} \delta(\boldsymbol{x}) .
$$

Now $\operatorname{det}(A)=\operatorname{det}\left(L \Lambda R^{\top} x\right)=\operatorname{det}(L) \operatorname{det}(\Lambda) \operatorname{det}\left(R^{\top}\right)$, it follows that

$$
|\operatorname{det}(A)|=|\operatorname{det}(L)||\operatorname{det}(\Lambda)|\left|\operatorname{det}\left(R^{\top}\right)\right|=\prod_{j=1}^{n} \lambda_{j}
$$

Therefore we get the desired identity: $\delta(A x)=\left|\operatorname{det}^{-1}(A)\right| \delta(x)$. This completes the proof.

Clearly, letting $A=a \mathbb{1}_{n}$ in the above gives Eq. (2.3).

\section{Dirac delta function of matrix argument}

Definition 3.1 (Dirac delta function of real-matrix argument). (i) For an $m \times n$ real matrix $X=$ $\left[x_{i j}\right] \in \mathbb{R}^{m \times n}$, the matrix delta function $\delta(X)$ is defined as

$$
\delta(X):=\prod_{i=1}^{m} \prod_{j=1}^{n} \delta\left(x_{i j}\right)
$$

In particular, the vector delta function is just a special case where $n=1$ in the matrix case.

(ii) For an $m \times m$ symmetric real matrix $X=\left[x_{i j}\right]$, the matrix delta function $\delta(X)$ is defined as

$$
\delta(X):=\prod_{i \leqslant j} \delta\left(x_{i j}\right)
$$


From the above definition, we see that the matrix delta function of a complex matrix is equal to the product of one-dimensional delta functions over the independent real and imaginary parts of this complex matrix. In view of this observation, we see that $\delta(X)=\delta(\operatorname{vec}(X))$, where vec $(X)$ is the vectorization of the matrix $X$. It is easily checked for a rectangular matrix. For the symmetric case, for example, take $2 \times 2$ symmetric real matrix $X=\left[\begin{array}{ll}x_{11} & x_{12} \\ x_{21} & x_{22}\end{array}\right]$ with $x_{12}=x_{21}$, then $\operatorname{vec}(X)=\left[x_{11}, x_{21}, x_{12}, x_{22}\right]^{\top}=\left[x_{11}, x_{12}, x_{12}, x_{22}\right]^{\top}$, thus $\operatorname{vec}(X)=x_{11}[1,0,0,0]^{\top}+x_{12}[0,1,1,0]^{\top}+$ $x_{22}[0,0,0,1]^{\top}$, i.e., there are three independent variables $\left\{x_{11}, x_{12}, x_{22}\right\}$ in the vector $\operatorname{vec}(X)$ just like in the matrix $X$, thus

$$
\delta(X)=\delta\left(x_{11}\right) \delta\left(x_{12}\right) \delta\left(x_{22}\right)=\delta(\operatorname{vec}(X))
$$

Proposition 3.2. For an $m \times m$ symmetric matrix $X$, we have

$$
\delta(X)=2^{-m} \pi^{-\frac{m(m+1)}{2}} \int e^{\mathrm{i} \operatorname{Tr}(T X)}[\mathrm{d} T]
$$

where $T=\left[t_{i j}\right]$ is also an $m \times m$ real symmetric matrix, and $[\mathrm{d} T]:=\prod_{i \leqslant j} \mathrm{~d} t_{i j}$.

Proof. Since

$$
\operatorname{Tr}(T X)=\sum_{j=1}^{m} t_{j j} x_{j j}+\sum_{i \neq j} t_{i j} x_{i j}=\sum_{j=1}^{m} t_{j j} x_{j j}+2 \sum_{i<j} t_{i j} x_{i j}
$$

implying that

$$
\begin{aligned}
\int e^{\mathrm{i} \operatorname{Tr}(T X)[\mathrm{d} T]} & =\prod_{j=1}^{m} \int \exp \left(\mathrm{i} t_{j j} x_{j j}\right) \mathrm{d} t_{j j} \prod_{1 \leqslant i<j \leqslant m} \int \exp \left(\mathrm{i} t_{i j}\left(2 x_{i j}\right)\right) \mathrm{d} t_{i j} \\
& =\prod_{j=1}^{m} 2 \pi \delta\left(x_{j j}\right) \times \prod_{1 \leqslant i<j \leqslant m} 2 \pi \delta\left(2 x_{i j}\right) \\
& =\prod_{j=1}^{m} 2 \pi \delta\left(x_{j j}\right) \times \prod_{1 \leqslant i<j \leqslant m} \pi \delta\left(x_{i j}\right)=2^{m} \pi^{(m+1)} \delta(X) .
\end{aligned}
$$

Therefore we get the desired identity.

Proposition 3.3. For $A \in \mathbb{R}^{m \times m}, B \in \mathbb{R}^{n \times n}$ and $X \in \mathbb{R}^{m \times n}$, we have

$$
\delta(A X B)=\left|\operatorname{det}^{-n}(A) \operatorname{det}^{-m}(B)\right| \delta(X) .
$$

Proof. We have already known that $\delta(A X B)=\delta(\operatorname{vec}(A X B))$. Since $\operatorname{vec}(A X B)=\left(A \otimes B^{\top}\right) \operatorname{vec}(X)$ [11], it follows that

$$
\begin{aligned}
\delta(A X B) & =\delta(\operatorname{vec}(A X B))=\delta\left(\left(A \otimes B^{\top}\right) \operatorname{vec}(X)\right) \\
& =\left|\operatorname{det}^{-1}\left(A \otimes B^{\top}\right)\right| \delta(\operatorname{vec}(X)) \\
& =\left|\operatorname{det}^{-n}(A) \operatorname{det}^{-m}(B)\right| \delta(X) .
\end{aligned}
$$

This completes the proof. 
Proposition 3.4. For $A \in \mathbb{R}^{n \times n}$ and $X=X^{\top} \in \mathbb{R}^{n \times n}$, we have

$$
\delta\left(A X A^{\top}\right)=|\operatorname{det}(A)|^{-(n+1)} \delta(X) .
$$

Proof. By using Eq. (3.3), it follows that

$$
\begin{aligned}
\delta\left(A X A^{\top}\right) & =2^{-n} \pi^{-\frac{n(n+1)}{2}} \int e^{\mathrm{i} \operatorname{Tr}\left(T A X A^{\top}\right)}[\mathrm{d} T] \\
& =2^{-n} \pi^{-\frac{n(n+1)}{2}} \int e^{\mathrm{i} \operatorname{Tr}\left(A^{\mathrm{T}} T A X\right)}[\mathrm{d} T] .
\end{aligned}
$$

Let $S=A^{\top} T A$. Then we have $[\mathrm{d} S]=|\operatorname{det}(A)|^{n+1}[\mathrm{~d} T]$ (see Proposition 2.8 in [11]). From this, we see that

$$
\delta\left(A X A^{\top}\right)=|\operatorname{det}(A)|^{-(n+1)} 2^{-n} \pi^{-\frac{n(n+1)}{2}} \int e^{\mathrm{i} \operatorname{Tr}(S X)}[\mathrm{d} S]=|\operatorname{det}(A)|^{-(n+1)} \delta(X) .
$$

This completes the proof.

Note that Dirac delta function for complex number is defined by $\delta(z):=\delta(\operatorname{Re}(z)) \delta(\operatorname{Im}(z))$, where $z=\operatorname{Re}(z)+\sqrt{-1} \operatorname{Im}(z)$ for $\operatorname{Re}(z), \operatorname{Im}(z) \in \mathbb{R}$. The complex number $z$ can be realized as a 2-dimensional real vector

$$
z \mapsto \widehat{z}:=\left[\begin{array}{c}
\operatorname{Re}(z) \\
\operatorname{Im}(z)
\end{array}\right]
$$

Thus

$$
\delta(z)=\delta(\widehat{z})=\delta\left(\left[\begin{array}{c}
\operatorname{Re}(z) \\
\operatorname{Im}(z)
\end{array}\right]\right)
$$

Then for $c \in \mathbb{C}, c z$ is represented as

$$
c z \mapsto\left[\begin{array}{c}
\operatorname{Re}(c) \operatorname{Re}(z)-\operatorname{Im}(c) \operatorname{Im}(z) \\
\operatorname{Im}(c) \operatorname{Re}(z)+\operatorname{Re}(c) \operatorname{Im}(z)
\end{array}\right]=\left[\begin{array}{cc}
\operatorname{Re}(c) & -\operatorname{Im}(c) \\
\operatorname{Im}(c) & \operatorname{Re}(c)
\end{array}\right]\left[\begin{array}{c}
\operatorname{Re}(z) \\
\operatorname{Im}(z)
\end{array}\right]
$$

we have

$$
\begin{aligned}
\delta(c z) & =\delta\left(\left[\begin{array}{cc}
\operatorname{Re}(c) & -\operatorname{Im}(c) \\
\operatorname{Im}(c) & \operatorname{Re}(c)
\end{array}\right]\left[\begin{array}{c}
\operatorname{Re}(z) \\
\operatorname{Im}(z)
\end{array}\right]\right) \\
& =\left|\operatorname{det}^{-1}\left(\left[\begin{array}{cc}
\operatorname{Re}(c) & -\operatorname{Im}(c) \\
\operatorname{Im}(c) & \operatorname{Re}(c)
\end{array}\right]\right)\right| \delta\left(\left[\begin{array}{c}
\operatorname{Re}(z) \\
\operatorname{Im}(z)
\end{array}\right]\right)=|c|^{-2} \delta(z) .
\end{aligned}
$$

Therefore we have

$$
\delta(c z)=|c|^{-2} \delta(z)
$$

Furthermore, if $z \in \mathbb{C}^{n}$, then

$$
\delta(c z)=|c|^{-2 n} \delta(z) .
$$


Proposition 3.5. For a full-ranked complex matrix $A \in \mathbb{C}^{n \times n}$, it holds that

$$
\delta(A z)=\frac{1}{\left|\operatorname{det}\left(A A^{*}\right)\right|} \delta(z)=|\operatorname{det}(A)|^{-2} \delta(z), \quad z \in \mathbb{C}^{n} .
$$

In particular, for any unitary matrix $U \in \mathrm{U}(n)$, we have

$$
\delta(U z)=\delta(z)
$$

Proof. Since $A z$ can be represented as

$$
\widehat{A} \widehat{z}=\left[\begin{array}{cc}
\operatorname{Re}(A) & -\operatorname{Im}(A) \\
\operatorname{Im}(A) & \operatorname{Re}(A)
\end{array}\right]\left[\begin{array}{c}
\operatorname{Re}(z) \\
\operatorname{Im}(z)
\end{array}\right]
$$

it follows that

$$
\begin{aligned}
\delta(A z) & =\delta\left(\left[\begin{array}{cc}
\operatorname{Re}(A) & -\operatorname{Im}(A) \\
\operatorname{Im}(A) & \operatorname{Re}(A)
\end{array}\right]\left[\begin{array}{c}
\operatorname{Re}(z) \\
\operatorname{Im}(z)
\end{array}\right]\right) \\
& =\left|\operatorname{det}^{-1}\left(\left[\begin{array}{cc}
\operatorname{Re}(A) & -\operatorname{Im}(A) \\
\operatorname{Im}(A) & \operatorname{Re}(A)
\end{array}\right]\right)\right| \delta\left(\left[\begin{array}{c}
\operatorname{Re}(z) \\
\operatorname{Im}(z)
\end{array}\right]\right) \\
& =\left|\operatorname{det}^{-1}\left(A A^{*}\right)\right| \delta(\boldsymbol{z}),
\end{aligned}
$$

where $\left|\operatorname{det}\left(\left[\begin{array}{cc}\operatorname{Re}(A) & -\operatorname{Im}(A) \\ \operatorname{Im}(A) & \operatorname{Re}(A)\end{array}\right]\right)\right|=\left|\operatorname{det}\left(A A^{*}\right)\right|$ can be found in [8, 11]. Therefore we have

$$
\delta(A z)=|\operatorname{det}(A)|^{-2} \delta(\boldsymbol{z}), \quad \boldsymbol{z} \in \mathbb{C}^{n} .
$$

If $A=U$ is a unitary matrix, then $|\operatorname{det}(U)|=1$. The desired result is obtained.

The second proof. Let $\widehat{A}=\left[\begin{array}{cc}\operatorname{Re}(A) & -\operatorname{Im}(A) \\ \operatorname{Im}(A) & \operatorname{Re}(A)\end{array}\right]$ and $\widehat{z}=\left[\begin{array}{c}\operatorname{Re}(z) \\ \operatorname{Im}(z)\end{array}\right]$. Then by Proposition 2.3,

$$
\delta(A z)=\delta(\widehat{A} \widehat{z})=\left|\operatorname{det}^{-1}(\widehat{A})\right| \delta(\widehat{z})=\left|\operatorname{det}^{-1}\left(A A^{*}\right)\right| \delta(z) .
$$

This completes the proof.

Definition 3.6 (Dirac delta function of complex-matrix argument). (i) For an $m \times n$ complex matrix $Z=\left[z_{i j}\right] \in \mathbb{C}^{m \times n}$, the matrix delta function $\delta(Z)$ is defined as

$$
\delta(Z):=\prod_{i=1}^{m} \prod_{j=1}^{n} \delta\left(\operatorname{Re}\left(z_{i j}\right)\right) \delta\left(\operatorname{Im}\left(z_{i j}\right)\right) .
$$

In particular, the vector delta function is just a special case where $n=1$ in the matrix case.

(ii) For an $m \times m$ Hermitian complex matrix $X=\left[x_{i j}\right] \in \mathbb{C}^{m \times m}$, the matrix delta function $\delta(X)$ is defined as

$$
\delta(X):=\prod_{j} \delta\left(x_{j j}\right) \prod_{i<j} \delta\left(\operatorname{Re}\left(x_{i j}\right)\right) \delta\left(\operatorname{Im}\left(x_{i j}\right)\right) .
$$


The Fourier integral representation of Dirac delta function can be extended to the matrix case. The following proposition is very important in this paper.

Proposition 3.7. For an $m \times m$ Hermitian complex matrix $X \in \mathbb{C}^{m \times m}$, we have

$$
\delta(X)=\frac{1}{2^{m} \pi^{m^{2}}} \int e^{\mathrm{i} \operatorname{Tr}(T X)}[\mathrm{d} T]
$$

where $T=\left[t_{i j}\right]$ is also an $m \times m$ Hermitian complex matrix, and $[\mathrm{d} T]:=\prod_{j} \mathrm{~d} t_{j j} \prod_{i<j} \mathrm{~d} \operatorname{Re}\left(t_{i j}\right) \mathrm{d} \operatorname{Im}\left(t_{i j}\right)$. Proof. Indeed, we know that

$$
\begin{aligned}
\operatorname{Tr}(T X) & =\sum_{j=1}^{m} t_{j j} x_{j j}+\sum_{i \neq j}\left(\bar{t}_{i j} x_{i j}\right)=\sum_{j=1}^{m} \operatorname{Re}\left(t_{j j}\right) \operatorname{Re}\left(x_{j j}\right)+\sum_{1 \leqslant i<j \leqslant m}\left(\bar{t}_{i j} x_{i j}+t_{i j} \bar{x}_{i j}\right) \\
& =\sum_{j=1}^{m} t_{j j} x_{j j}+\sum_{1 \leqslant i<j \leqslant m} 2\left(\operatorname{Re}\left(t_{i j}\right) \operatorname{Re}\left(x_{i j}\right)+\operatorname{Im}\left(t_{i j}\right) \operatorname{Im}\left(x_{i j}\right)\right)
\end{aligned}
$$

implying that

$$
\begin{aligned}
\int e^{\mathrm{i} \operatorname{Tr}(T X)[\mathrm{d} T]=} & \prod_{j=1}^{m} \int \exp \left(\mathrm{i} t_{j j} x_{j j}\right) \mathrm{d} t_{j j} \\
& \times \prod_{1 \leqslant i<j \leqslant m} \int \exp \left(\operatorname{iRe}\left(t_{i j}\right)\left(2 \operatorname{Re}\left(x_{i j}\right)\right)\right) \mathrm{d} \operatorname{Re}\left(t_{i j}\right) \\
& \times \prod_{1 \leqslant i<j \leqslant m} \int \exp \left(\operatorname{iIm}\left(t_{i j}\right)\left(2 \operatorname{Im}\left(x_{i j}\right)\right)\right) \mathrm{d} \operatorname{Im}\left(t_{i j}\right) \\
= & \prod_{j=1}^{m} 2 \pi \delta\left(x_{j j}\right) \times \prod_{1 \leqslant i<j \leqslant m} 2 \pi \delta\left(2 \operatorname{Re}\left(x_{i j}\right)\right) 2 \pi \delta\left(2 \operatorname{Im}\left(x_{i j}\right)\right) \\
= & \prod_{j=1}^{m} 2 \pi \delta\left(x_{j j}\right) \times \prod_{1 \leqslant i<j \leqslant m} \pi \delta\left(\operatorname{Re}\left(x_{i j}\right)\right) \pi \delta\left(\operatorname{Im}\left(x_{i j}\right)\right) \\
= & (2 \pi)^{m}\left(\pi^{2}\right)^{\left(\begin{array}{c}
m \\
2
\end{array}\right)} \prod_{j} \delta\left(x_{j j}\right) \prod_{i<j} \delta\left(\operatorname{Re}\left(x_{i j}\right)\right) \delta\left(\operatorname{Im}\left(x_{i j}\right)\right) \\
= & 2^{m} \pi^{m^{2}} \delta(X) .
\end{aligned}
$$

Therefore we get the desired identity.

Remark 3.8. Indeed, since

$$
\operatorname{Tr}\left(T^{\text {off }} X^{\text {off }}\right)=\sum_{i<j} 2\left(\operatorname{Re}\left(t_{i j}\right) \operatorname{Re}\left(x_{i j}\right)+\operatorname{Im}\left(t_{i j}\right) \operatorname{Im}\left(x_{i j}\right)\right)
$$

and

$$
\left[\mathrm{d} T^{\mathrm{off}}\right]=\prod_{i<j} \mathrm{~d} \operatorname{Re}\left(t_{i j}\right) \mathrm{d} \operatorname{Im}\left(t_{i j}\right)
$$


it follows that

$$
\begin{aligned}
& \int\left[\mathrm{d} T^{\text {off }}\right] \exp \left(\mathrm{i} \operatorname{Tr}\left(T^{\text {off }} X^{\text {off }}\right)\right) \\
& =\prod_{i<j} \int \mathrm{d} \operatorname{Re}\left(t_{i j}\right) \exp \left(i \operatorname{Re}\left(t_{i j}\right)\left(2 \operatorname{Re}\left(x_{i j}\right)\right)\right) \int \mathrm{d} \operatorname{Im}\left(t_{i j}\right) \exp \left(\operatorname{iIm}\left(t_{i j}\right)\left(2 \operatorname{Im}\left(x_{i j}\right)\right)\right) \\
& =\prod_{i<j} 2 \pi \delta\left(2 \operatorname{Re}\left(x_{i j}\right)\right) \cdot 2 \pi \delta\left(2 \operatorname{Im}\left(x_{i j}\right)\right)=\prod_{i<j} \pi \delta\left(\operatorname{Re}\left(x_{i j}\right)\right) \pi \delta\left(\operatorname{Im}\left(x_{i j}\right)\right) \\
& =\pi^{2\left(\begin{array}{c}
m \\
2
\end{array}\right)} \prod_{i<j} \delta\left(\operatorname{Re}\left(x_{i j}\right)\right) \delta\left(\operatorname{Im}\left(x_{i j}\right)\right)=\pi^{m(m-1)} \delta\left(X^{\text {off }}\right) .
\end{aligned}
$$

From the above discussion, we see that (3.19) can be separated into two identities below:

$$
\begin{aligned}
\delta\left(X^{\text {diag }}\right) & =\frac{1}{(2 \pi)^{m}} \int\left[\mathrm{d} T^{\text {diag }}\right] e^{\mathrm{i} \operatorname{Tr}\left(T^{\text {diag }} X^{\text {diag }}\right)}, \\
\delta\left(X^{\text {off }}\right) & =\frac{1}{\pi^{m(m-1)}} \int\left[\mathrm{d} T^{\text {off }}\right] e^{\mathrm{i} \operatorname{Tr}\left(T^{\text {off }} X^{\text {off }}\right)} .
\end{aligned}
$$

Note that the identity in Proposition 3.7 is used in deriving the joint distribution of diagonal part of Wishart matrix ensemble [12], and it is also used in obtaining derivative principle for unitarily invariant random matrix ensemble in [9]. More generally, the fact can be found in [2] that the derivative principle for invariant measure is used to investigate the joint distribution of eigenvalues of local states from the same multipartite pure states.

Proposition 3.9. For $A \in \mathbb{C}^{m \times m}$ and $B \in \mathbb{C}^{n \times n}$, let $Z \in \mathbb{C}^{m \times n}$. Then we have

$$
\delta(A Z B)=\operatorname{det}^{-n}\left(A A^{*}\right) \operatorname{det}^{-m}\left(B B^{*}\right) \delta(Z) .
$$

Proof. Now $A Z B$ can be represented as, via $\widehat{X Y}=\widehat{X} \widehat{Y}$,

$$
\widehat{A Z B}=\widehat{A} \widehat{Z} \widehat{B}=\left[\begin{array}{cc}
\operatorname{Re}(A) & -\operatorname{Im}(A) \\
\operatorname{Im}(A) & \operatorname{Re}(A)
\end{array}\right]\left[\begin{array}{cc}
\operatorname{Re}(Z) & -\operatorname{Im}(Z) \\
\operatorname{Im}(Z) & \operatorname{Re}(Z)
\end{array}\right]\left[\begin{array}{cc}
\operatorname{Re}(B) & -\operatorname{Im}(B) \\
\operatorname{Im}(B) & \operatorname{Re}(B)
\end{array}\right]
$$

Then from Eq. (3.4), we see that

$$
\begin{aligned}
\delta(A Z B) & =\delta(\widehat{A Z B})=\delta(\widehat{A} \widehat{Z} \widehat{B})=\left|\operatorname{det}^{-n}(\widehat{A}) \operatorname{det}^{-m}(\widehat{B})\right| \delta(\widehat{Z}) \\
& =\operatorname{det}^{-n}\left(A A^{*}\right) \operatorname{det}^{-m}\left(B B^{*}\right) \delta(Z) .
\end{aligned}
$$

The result is proven.

Proposition 3.10. For $A \in \mathbb{C}^{m \times m}$, and $m \times m$ Hermitian complex matrix $X \in \mathbb{C}^{m \times m}$, we have

$$
\delta\left(A X A^{*}\right)=\left|\operatorname{det}\left(A A^{*}\right)\right|^{-m} \delta(X)
$$


Proof. By using the Fourier transform of the matrix delta function (see Eq. (3.19))

$$
\delta\left(A X A^{*}\right)=\frac{1}{2^{m} \pi^{m^{2}}} \int e^{\mathrm{i} \operatorname{Tr}\left(T A X A^{*}\right)}[\mathrm{d} T]=\frac{1}{2^{m} \pi^{m^{2}}} \int e^{\mathrm{i} \operatorname{Tr}\left(A^{*} T A X\right)}[\mathrm{d} T],
$$

where $T=\left[t_{i j}\right]$ is also an $m \times m$ Hermitian complex matrix, and $[\mathrm{d} T]:=\prod_{j} \mathrm{~d} t_{j j} \prod_{i<j} \mathrm{~d} \operatorname{Re}\left(t_{i j}\right) \mathrm{d} \operatorname{Im}\left(t_{i j}\right)$.

Let $H=A^{*} T A$. Then we have (see Proposition 3.4 in [11]):

$$
[\mathrm{d} H]=\left|\operatorname{det}\left(A A^{*}\right)\right|^{m}[\mathrm{~d} T] \text {. }
$$

Thus

$$
\delta\left(A X A^{*}\right)=\left|\operatorname{det}\left(A A^{*}\right)\right|^{-m} \frac{1}{2^{m} \pi^{m^{2}}} \int e^{\mathrm{i} \operatorname{Tr}(H X)}[\mathrm{d} H]=\left|\operatorname{det}\left(A A^{*}\right)\right|^{-m} \delta(X),
$$

We are done.

\section{Applications}

\subsection{Joint distribution of eigenvalues of Wishart matrix ensemble}

The first application is to calculate the joint distribution of Wishart matrix ensemble [6]. Note that the so-called Wishart matrix ensemble is the set of all complex matrices of the form $W=Z Z^{+}$ with $Z$ an $m \times n(m \leqslant n)$ complex Gaussian matrix, i.e., a matrix with all entries being standard complex Gaussian random variables,

$$
\varphi(Z)=\frac{1}{\pi^{m n}} \exp \left(-\operatorname{Tr}\left(Z Z^{\dagger}\right)\right),
$$

the distribution density of $W$ is given by

$$
\begin{aligned}
\mathbf{P}(W) & =\int \delta\left(W-Z Z^{\dagger}\right) \varphi(Z)[\mathrm{d} Z] \\
& =\frac{1}{\pi^{m n}} \int \delta\left(W-Z Z^{\dagger}\right) \exp \left(-\operatorname{Tr}\left(Z Z^{\dagger}\right)\right)[\mathrm{d} Z] .
\end{aligned}
$$

That is,

$$
\mathbf{P}(W)=\frac{1}{\pi^{m n}} e^{-\operatorname{Tr}(W)} \int \delta\left(W-Z Z^{\dagger}\right)[\mathrm{d} Z] .
$$

Let $Z=\sqrt{W} Y$. Then $Z Z^{+}=\sqrt{W} Y Y^{\dagger} \sqrt{W}$ and $[\mathrm{d} Z]=\operatorname{det}(W)^{n}[\mathrm{~d} Y]$. By Proposition 3.10, we get

$$
\delta\left(W-Z Z^{\dagger}\right)=\delta\left(\sqrt{W}\left(\mathbb{1}-Y Y^{\dagger}\right) \sqrt{W}\right)=\frac{1}{\operatorname{det}(W)^{m}} \delta\left(\mathbb{1}-Y Y^{\dagger}\right) .
$$

Therefore

$$
\mathbf{P}(W)=\frac{1}{\pi^{m n}} \operatorname{det}^{n-m}(W) e^{-\operatorname{Tr}(W)} \int \delta\left(\mathbb{1}_{m}-Y Y^{\dagger}\right)[\mathrm{d} Y] \propto \operatorname{det}^{n-m}(W) e^{-\operatorname{Tr}(W)},
$$

where $\int \delta\left(\mathbb{1}-Y Y^{+}\right)[\mathrm{d} Y]:=C$ is a constant, independent of $W$. 
Proposition 4.1. It holds that

$$
\int \delta\left(\mathbb{1}_{m}-Y Y^{\dagger}\right)[\mathrm{d} Y]=\frac{\pi^{\frac{1}{2} m(2 n-m+1)}}{\prod_{k=1}^{m}(n-k) !}
$$

In particular, (i) for $m=1$,

$$
\int \delta(1-\langle\boldsymbol{y}, \boldsymbol{y}\rangle)[\mathrm{d} \boldsymbol{y}]=\frac{\pi^{n}}{(n-1) !}=\frac{\pi^{n}}{\Gamma(n)} .
$$

(ii) for $m=n$, we have

$$
\int \delta\left(\mathbb{1}_{n}-Y Y^{\dagger}\right)[\mathrm{d} Y]=\frac{\pi^{\frac{1}{2} n(n+1)}}{\prod_{k=1}^{n} \Gamma(k)}=2^{-n} \cdot \operatorname{vol}(\mathrm{U}(n)) .
$$

Proof. Since

$$
1=\int \mathbf{P}(W)[\mathrm{d} W]=\frac{C}{\pi^{m n}} \int_{W>0} \operatorname{det}^{n-m}(W) e^{-\operatorname{Tr}(W)}[\mathrm{d} W],
$$

where via $W=U w U^{\dagger}$ for $w=\operatorname{diag}\left(w_{1}, \ldots, w_{m}\right)$ where $w_{1}>\cdots>w_{m}$

$$
[\mathrm{d} W]=\Delta(w)^{2}[\mathrm{~d} w]\left[U^{\dagger} \mathrm{d} U\right]
$$

It follows that

$$
1=\frac{C}{\pi^{m n}} \operatorname{vol}\left(\mathrm{U}(m) / \mathbb{T}^{m}\right) \int_{w_{1}>\cdots>w_{m}} \Delta(w)^{2} \operatorname{det}^{n-m}(w) e^{-\operatorname{Tr}(w)}[\mathrm{d} w] .
$$

By using Selberg integral formula, we see that

$$
\int \Delta(w)^{2} \operatorname{det}^{n-m}(w) e^{-\operatorname{Tr}(w)}[\mathrm{d} w]=\prod_{k=1}^{m} k !(n-k) !
$$

Thus

$$
\int_{w_{1}>\cdots>w_{m}} \Delta(w)^{2} \operatorname{det}^{n-m}(w) e^{-\operatorname{Tr}(w)}[\mathrm{d} w]=\frac{1}{m !} \prod_{k=1}^{m} k !(n-k) ! .
$$

We conclude that

$$
\int \delta\left(\mathbb{1}-Y Y^{\dagger}\right)[\mathrm{d} Y]=\frac{\operatorname{vol}\left(\mathbb{T}^{m}\right) m ! \pi^{m n}}{\operatorname{vol}(\mathrm{U}(m)) \prod_{k=1}^{m} k !(n-k) !} .
$$

Now

$$
\operatorname{vol}(\mathrm{U}(m))=\frac{2^{m} \pi^{\frac{m(m+1)}{2}}}{\prod_{k=1}^{m} \Gamma(k)} \quad \text { and } \quad \operatorname{vol}\left(\mathbb{T}^{m}\right)=(2 \pi)^{m}
$$

We obtain that

$$
\int \delta\left(\mathbb{1}-Y Y^{\dagger}\right)[\mathrm{d} Y]=\frac{\pi^{\frac{1}{2} m(2 n-m+1)}}{\prod_{k=1}^{m}(n-k) !} .
$$

This completes the proof. 
Corollary 4.2. It holds that

$$
\int \delta\left(W-Z Z^{\dagger}\right)[\mathrm{d} Z]=\frac{\pi^{\frac{1}{2} m(2 n-m+1)}}{\prod_{k=1}^{m}(n-k) !} \operatorname{det}(W)^{n-m} .
$$

Proof. It is easily seen that

$$
\begin{aligned}
\int \delta\left(W-Z Z^{+}\right)[\mathrm{d} Z] & =\operatorname{det}(W)^{n-m} \int \delta\left(\mathbb{1}_{m}-Y Y^{\dagger}\right)[\mathrm{d} Y] \\
& =\frac{\pi^{\frac{1}{2} m(2 n-m+1)}}{\prod_{k=1}^{m}(n-k) !} \operatorname{det}(W)^{n-m}
\end{aligned}
$$

Here we used the result in Proposition 4.1.

Remark 4.3. Denote $U(m, n):=\left\{Z \in \mathbb{C}^{m \times n}: Z Z^{+}=\mathbb{1}_{m}\right\}(m \leqslant n)$. Note that

$$
\operatorname{vol}(\mathrm{U}(m, n))=\int \delta\left(\mathbb{1}_{m}-\mathrm{ZZ} \mathrm{Z}^{\dagger}\right)\left[\mathrm{Z}^{\dagger} \mathrm{d} Z\right]=\frac{2^{m} \pi^{\frac{1}{2} m(2 n-m+1)}}{\prod_{k=1}^{m}(n-k) !} .
$$

This indicates that

$$
\int \delta\left(\mathbb{1}_{m}-Z Z^{\dagger}\right)\left[Z^{\dagger} \mathrm{d} Z\right]=2^{m} \int \delta\left(\mathbb{1}_{m}-Z Z^{\dagger}\right)[\mathrm{d} Z] .
$$

In addition, the delta integral can be reformulated in terms of another form:

$$
\int \delta\left(W-Z Z^{+}\right)[\mathrm{dZ}]=\int_{Z^{+}=W}[\mathrm{dZ}] .
$$

Finally, we have seen that

$$
\mathbf{P}(W)=\frac{\operatorname{det}(W)^{n-m} e^{-\operatorname{Tr}(W)}}{\pi^{\left(\begin{array}{c}
m \\
2
\end{array}\right)} \prod_{k=1}^{m}(n-k) !} .
$$

\subsection{Joint distribution of eigenvalues of induced random quantum state ensemble}

Any mixed state $\rho$ (i.e., nonnegative complex matrix of trace-one) acting on $\mathcal{H}_{m}$, may be purified by finding a pure state $|X\rangle$ in the composite Hilbert space $\mathcal{H}_{m} \otimes \mathcal{H}_{m}$, such that $\rho$ is given by the partial tracing over the auxiliary subsystem,

$$
|X\rangle \longrightarrow \rho=\operatorname{Tr}_{2}(|X\rangle\langle X|) .
$$

In a loose sense, the purification corresponds to treating any density matrix of size $m$ as a vector of size $m^{2}$.

Consider a bipartite $m \otimes n$ composite quantum system. Pure states of this system $|X\rangle$ may be represented by a rectangular complex matrix $X$. The partial tracing with respect to the $n$ dimensional subspace gives a reduced density matrix of size $m: \rho=\operatorname{Tr}_{n}(|X\rangle\langle X|)$. The natural measure in the space of $m n$-dimensional pure states induces the measure $P_{m, n}(\rho)$ in the space of the reduced density matrices of size $m$. 
Without loss of generality, we assume that $m \leqslant n$, then $\rho$ is generically positive definite (here something is generic means it holds with probability one). In any case, we are only interested in the distribution of the positive eigenvalues. Let us call the corresponding positive reduced density matrix again $\rho=X X^{\dagger}$, where $X$ is a $m \times n$ matrix. First we calculate the distribution of matrix elements

$$
\mathbf{P}(\rho) \propto \int[\mathrm{d} X] \delta\left(\rho-X X^{\dagger}\right) \delta\left(1-\operatorname{Tr}\left(X X^{\dagger}\right)\right)
$$

where the first delta function is a delta function of a Hermitian matrix and in the second delta function $\operatorname{Tr}\left(X X^{\dagger}\right)$ may be substituted by $\operatorname{Tr}(\rho)$. Since $\rho$ is positive definite we can make a transformation

$$
X=\sqrt{\rho} \widetilde{X}
$$

it follows that $[\mathrm{dX}]=(\operatorname{det} \rho)^{n}[\mathrm{~d} \widetilde{X}][11]$. The matrix delta function may now be written as

$$
\delta\left(\sqrt{\rho}\left(\mathbb{1}-\widetilde{X} \widetilde{X}^{\dagger}\right) \sqrt{\rho}\right)=(\operatorname{det} \rho)^{-m} \delta\left(\mathbb{1}-\widetilde{X} \widetilde{X}^{\dagger}\right) .
$$

As the result the distribution of matrix elements is given by

$$
\mathbf{P}(\rho) \propto \theta(\rho) \delta(1-\operatorname{Tr}(\rho))(\operatorname{det} \rho)^{n-m}
$$

where the theta function assures that $\rho$ is positive definite. It is then easy to show by the methods of random matrix theory that the joint density of eigenvalues $\Lambda=\left\{\lambda_{1}, \ldots, \lambda_{m}\right\}$ of $\rho$ is given by

$$
\mathbf{P}_{m, n}\left(\lambda_{1}, \ldots, \lambda_{m}\right) \propto \delta\left(1-\sum_{j=1}^{m} \lambda_{j}\right) \prod_{j=1}^{m} \lambda_{j}^{n-m} \theta\left(\lambda_{j}\right) \prod_{1 \leqslant i<j \leqslant m}\left(\lambda_{i}-\lambda_{j}\right)^{2} .
$$

This result is firstly obtained by Życzkowski [13] in 2001.

\subsection{Joint distribution of eigenvalues of two Wishart matrices}

The second application is to calculate the distribution of the sum of a finite number of complex Wishart matrices taken from the same Wishart ensemble [6]. The distribution of the sum of two Wishart matrices is considered in [7]. Let us consider two independent complex matrices $A$ and $B$ of dimensions $m \times n_{A}$ and $m \times n_{B}$ taken, respectively, from the distributions

$$
\begin{aligned}
& \mathbf{P}_{A}(A)=\frac{1}{\pi^{m n_{A}} \operatorname{det}^{n_{A}}\left(\Sigma_{A}\right)} \exp \left(-\operatorname{Tr}\left(\Sigma_{A}^{-1} A A^{\dagger}\right)\right), \\
& \mathbf{P}_{B}(B)=\frac{1}{\pi^{m n_{B}} \operatorname{det}^{n_{B}}\left(\Sigma_{B}\right)} \exp \left(-\operatorname{Tr}\left(\Sigma_{B}^{-1} B B^{\dagger}\right)\right) .
\end{aligned}
$$


Here $\Sigma_{A}, \Sigma_{B}$ are the covariance matrices. Since the domains of $A$ and $B$ remain invariant under unitary rotation, without loss of generality, we may take $\Sigma_{A}=\operatorname{diag}\left(\sigma_{A 1}, \ldots, \sigma_{A m}\right)$ and $\Sigma_{B}=$ $\operatorname{diag}\left(\sigma_{B 1}, \ldots, \sigma_{B m}\right)$. We assume that $m \leqslant n_{A}, n_{B}$. And we have

$$
\int[\mathrm{d} A] \mathbf{P}_{A}(A)=1 \text { and } \int[\mathrm{d} B] \mathbf{P}_{B}(B)=1 .
$$

The matrix $A A^{\dagger}$ and $B B^{\dagger}$ are then $n$-variate complex-Wishart-distributed, i.e., $A A^{\dagger} \sim W_{m}^{\mathrm{C}}\left(n_{A}, \Sigma_{A}\right)$ and $B B^{\dagger} \sim W_{m}^{\mathbb{C}}\left(n_{B}, \Sigma_{B}\right)$.

Ones are interested in the statistics of the ensemble of $m \times m$ Hermitian matrices

$$
W=A A^{\dagger}+B B^{\dagger}
$$

The distribution of $W$ can be obtained as

$$
\mathbf{P}_{W}(W)=\int[\mathrm{d} A] \int[\mathrm{d} B] \delta\left(W-A A^{\dagger}-B B^{\dagger}\right) \mathbf{P}_{A}(A) \mathbf{P}_{B}(B) .
$$

In what follows, our method will be different from Kumar's in [7]. Let $A=\sqrt{W} \widetilde{A}$ and $B=\sqrt{W} \widetilde{B}$. Then $[\mathrm{d} A]=\operatorname{det}(W)^{n_{A}}[\mathrm{~d} \widetilde{A}]$ and $[\mathrm{d} B]=\operatorname{det}(W)^{n_{B}}[\mathrm{~d} \widetilde{B}]$. We also have

$$
\delta\left(W-A A^{\dagger}-B B^{\dagger}\right)=\operatorname{det}(W)^{-m} \delta\left(\mathbb{1}-\widetilde{A} \widetilde{A}^{\dagger}-\widetilde{B} \widetilde{B}^{\dagger}\right) .
$$

Then

$$
\begin{aligned}
\mathbf{P}_{W}(W)= & \frac{\operatorname{det}^{n_{A}+n_{B}-m}(W)}{\pi^{m\left(n_{A}+n_{B}\right)} \operatorname{det}^{n_{A}}\left(\Sigma_{A}\right) \operatorname{det}^{n_{B}}\left(\Sigma_{B}\right)} \int[\mathrm{d} \widetilde{A}] \int[\mathrm{d} \widetilde{B}] \\
& \times \delta\left(\mathbb{1}-\widetilde{A} \widetilde{A}^{\dagger}-\widetilde{B} \widetilde{B}^{\dagger}\right) e^{-\operatorname{Tr}\left(\sqrt{W} \Sigma_{A}^{-1} \sqrt{W} \widetilde{A} \widetilde{A}^{+}\right)-\operatorname{Tr}\left(\sqrt{W} \Sigma_{B}^{-1} \sqrt{W} \widetilde{B} \widetilde{B}^{+}\right)} .
\end{aligned}
$$

That is,

$$
\begin{aligned}
\mathbf{P}_{W}(W) \propto & \operatorname{det}^{n_{A}+n_{B}-m}(W) \\
& \times \int[\mathrm{d} \widetilde{A}] \int[\mathrm{d} \widetilde{B}] \delta\left(\mathbb{1}-\widetilde{A} \widetilde{A}^{+}-\widetilde{B} \widetilde{B}^{\dagger}\right) e^{-\operatorname{Tr}\left(\sqrt{W} \Sigma_{A}^{-1} \sqrt{W} \widetilde{A} \widetilde{A}^{\dagger}+\sqrt{W} \Sigma_{B}^{-1} \sqrt{W} \widetilde{B} \widetilde{B}^{\dagger}\right)} .
\end{aligned}
$$

Now consider the reduction of the sum $\operatorname{Tr}\left(\sqrt{W} \Sigma_{A}^{-1} \sqrt{W} \widetilde{A} \widetilde{A}^{\dagger}+\sqrt{W} \Sigma_{B}^{-1} \sqrt{W} \widetilde{B} \widetilde{B}^{\dagger}\right)$. Since $\mathbb{1}=$ $\widetilde{A} \widetilde{A}^{\dagger}+\widetilde{B} \widetilde{B}^{\dagger}$, it follows that

$$
\begin{aligned}
& \operatorname{Tr}\left(\sqrt{W} \Sigma_{A}^{-1} \sqrt{W} \widetilde{A} \widetilde{A}^{\dagger}\right)+\operatorname{Tr}\left(\sqrt{W} \Sigma_{B}^{-1} \sqrt{W} \widetilde{B} \widetilde{B}^{\dagger}\right) \\
& =\operatorname{Tr}\left(W \Sigma_{A}^{-1}\right)+\operatorname{Tr}\left(\sqrt{W}\left(\Sigma_{B}^{-1}-\Sigma_{A}^{-1}\right) \sqrt{W} \widetilde{B} \widetilde{B}^{\dagger}\right) \\
& =\operatorname{Tr}\left(W \Sigma_{B}^{-1}\right)+\operatorname{Tr}\left(\sqrt{W}\left(\Sigma_{A}^{-1}-\Sigma_{B}^{-1}\right) \sqrt{W} \widetilde{A} \widetilde{A}^{+}\right) .
\end{aligned}
$$

Hence

$$
\mathbf{P}_{W}(W) \propto \frac{\operatorname{det}^{n_{A}+n_{B}-m}(W)}{e^{\operatorname{Tr}\left(W \Sigma_{A}^{-1}\right)}} Q(W),
$$


where

$$
\begin{aligned}
Q(W) & :=\int[\mathrm{d} \widetilde{A}] \int[\mathrm{d} \widetilde{B}] \delta\left(\mathbb{1}-\widetilde{A} \widetilde{A}^{\dagger}-\widetilde{B} \widetilde{B}^{\dagger}\right) e^{\operatorname{Tr}\left(\sqrt{W}\left(\Sigma_{A}^{-1}-\Sigma_{B}^{-1}\right) \sqrt{W} \widetilde{B} \widetilde{B}^{\dagger}\right)} \\
& =\int_{0<\widetilde{A} \widetilde{A}^{\dagger}<\mathbb{1}_{m}}[\mathrm{~d} \widetilde{A}] e^{\operatorname{Tr}\left(\sqrt{W}\left(\Sigma_{A}^{-1}-\Sigma_{B}^{-1}\right) \sqrt{W}\left(\mathbb{1}-\widetilde{A} \widetilde{A}^{\dagger}\right)\right)} \\
& =e^{\operatorname{Tr}\left(W\left(\Sigma_{A}^{-1}-\Sigma_{B}^{-1}\right)\right)} \int_{0<\widetilde{A} \widetilde{A}^{\dagger}<\mathbb{1}_{m}}[\mathrm{~d} \widetilde{A}] e^{\operatorname{Tr}\left(\sqrt{W}\left(\Sigma_{B}^{-1}-\Sigma_{A}^{-1}\right) \sqrt{W} \widetilde{A} \widetilde{A}^{\dagger}\right)}
\end{aligned}
$$

That is,

$$
\mathbf{P}_{W}(W) \propto \frac{\operatorname{det}^{n_{A}+n_{B}-m}(W)}{e^{\operatorname{Tr}\left(W \Sigma_{B}^{-1}\right)}} \int_{0<\widetilde{A} \widetilde{A}^{\dagger}<\mathbb{1}_{m}}[\mathrm{~d} \widetilde{A}] e^{\operatorname{Tr}\left(\sqrt{W}\left(\Sigma_{B}^{-1}-\Sigma_{A}^{-1}\right) \sqrt{W} \widetilde{A} \widetilde{A}^{+}\right)} .
$$

Similarly, we have

$$
\mathbf{P}_{W}(W) \propto \frac{\operatorname{det}^{n_{A}+n_{B}-m}(W)}{e^{\operatorname{Tr}\left(W \Sigma_{A}^{-1}\right)}} \int_{0<\widetilde{B}^{\dagger}<\mathbb{1}_{m}}[\mathrm{~d} \widetilde{B}] e^{\operatorname{Tr}\left(\sqrt{W}\left(\Sigma_{A}^{-1}-\Sigma_{B}^{-1}\right) \sqrt{W} \widetilde{B}^{+}\right)} .
$$

In fact, we have

$$
\begin{aligned}
& \frac{1}{e^{\operatorname{Tr}\left(W \Sigma_{B}^{-1}\right)}} \int_{0<\widetilde{A} \widetilde{A}^{\dagger}<\mathbb{1}_{m}}[\mathrm{~d} \widetilde{A}] e^{\operatorname{Tr}\left(\sqrt{W}\left(\Sigma_{B}^{-1}-\Sigma_{A}^{-1}\right) \sqrt{W} \widetilde{A} \widetilde{A}^{+}\right)} \\
& =\frac{1}{e^{\operatorname{Tr}\left(W \Sigma_{A}^{-1}\right)}} \int_{0<\widetilde{B} \widetilde{B}^{\dagger}<\mathbb{1}_{m}}[\mathrm{~d} \widetilde{B}] e^{\operatorname{Tr}\left(\sqrt{W}\left(\Sigma_{A}^{-1}-\Sigma_{B}^{-1}\right) \sqrt{W} \widetilde{B} \widetilde{B}^{\dagger}\right)} .
\end{aligned}
$$

Let $\Lambda=\sqrt{W}\left(\Sigma_{A}^{-1}-\Sigma_{B}^{-1}\right) \sqrt{W}$. By Gram-Schmidt orthogonalization procedure to write $\widetilde{A}=T U_{1}$, where $U_{1}$ is $m \times n_{A}$ matrix such that $U_{1} U_{1}^{+}=\mathbb{1}_{m}$ and $T$ is a $m \times m$ lower triangular matrix with diagonal entries real and positive. Then

$$
[\mathrm{d} \widetilde{A}]=\prod_{j=1}^{m} t_{j j}^{2\left(n_{A}-j\right)+1}[\mathrm{~d} T]\left[\mathrm{d} U_{1} U^{\dagger}\right],
$$

where $U$ is the enlarged $n_{A} \times n_{A}$ unitary matrix of $U_{1}$. Let $X=\widetilde{A} \widetilde{A}^{\dagger}=T T^{\dagger}$. We then have [11]:

$$
[\mathrm{d} X]=2^{m} \prod_{j=1}^{m} t_{j j}^{2(m-j)+1}[\mathrm{~d} T] .
$$

Combining together the above results gives that [11]

$$
[\mathrm{d} \widetilde{A}]=2^{-m} \operatorname{det}^{n_{A}-m}(X)[\mathrm{d} X]\left[\mathrm{d} U_{1} U^{\dagger}\right] .
$$

This means that

$$
\begin{aligned}
\int_{0<\widetilde{A} \widetilde{A}^{\dagger}<\mathbb{1}_{m}}[\mathrm{~d} \widetilde{A}] e^{\operatorname{Tr}\left(\Lambda \widetilde{A} \widetilde{A}^{\dagger}\right)} & =2^{-m} \int_{0}^{\mathbb{1}_{m}}[\mathrm{~d} X] \operatorname{det}^{n_{A}-m}(X) e^{\operatorname{Tr}(\Lambda X)} \int\left[\mathrm{d} U_{1} U^{\dagger}\right] \\
& \propto \int_{0}^{\mathbb{1}_{m}}[\mathrm{~d} X] \operatorname{det}^{n_{A}-m}(X) e^{\operatorname{Tr}(\Lambda X)} .
\end{aligned}
$$

Similarly,

$$
\int_{0<\widetilde{B} \widetilde{B}^{\dagger}<\mathbb{1}_{m}}[\mathrm{~d} \widetilde{B}] e^{-\operatorname{Tr}\left(\Lambda \widetilde{B} \widetilde{B}^{+}\right)} \propto \int_{0}^{\mathbb{1}_{m}}[\mathrm{~d} X] \operatorname{det}^{n_{B}-m}(X) e^{-\operatorname{Tr}(\Lambda X)}
$$


Theorem 4.4. It holds that

$$
\mathbf{P}_{W}(W) \propto \frac{\operatorname{det}^{n_{A}+n_{B}-m}(W)}{e^{\operatorname{Tr}\left(W \Sigma_{B}^{-1}\right)}} \int_{0}^{\mathbb{1}_{m}}[\mathrm{~d} X] \operatorname{det}^{n_{A}-m}(X) e^{\operatorname{Tr}(\Lambda X)}
$$

and

$$
\mathbf{P}_{W}(W) \propto \frac{\operatorname{det}^{n_{A}+n_{B}-m}(W)}{e^{\operatorname{Tr}\left(W \Sigma_{A}^{-1}\right)}} \int_{0}^{\mathbb{1}_{m}}[\mathrm{~d} X] \operatorname{det}^{n_{B}-m}(X) e^{-\operatorname{Tr}(\Lambda X)} .
$$

Moreover

$$
\frac{1}{e^{\operatorname{Tr}\left(W \Sigma_{B}^{-1}\right)}} \int_{0}^{\mathbb{1}_{m}}[\mathrm{~d} X] \operatorname{det}^{n_{A}-m}(X) e^{\operatorname{Tr}(\Lambda X)}=\frac{1}{e^{\operatorname{Tr}\left(W \Sigma_{A}^{-1}\right)}} \int_{0}^{\mathbb{1}_{m}}[\mathrm{~d} X] \operatorname{det}^{n_{B}-m}(X) e^{-\operatorname{Tr}(\Lambda X)} .
$$

In particular, if $\Lambda=0$ (i.e., $\Sigma_{A}=\Sigma_{B}:=\Sigma$ ), then

$$
\mathbf{P}_{W}(W) \propto \frac{\operatorname{det}^{n_{A}+n_{B}-m}(W)}{e^{\operatorname{Tr}\left(W \Sigma^{-1}\right)}} .
$$

In the following, we calculate, via $X=U \operatorname{diag}(x) U^{\dagger}$ where $\operatorname{diag}(x)=\operatorname{diag}\left(x_{1}, \ldots, x_{m}\right)$,

$$
\int_{0}^{\mathbb{1}_{m}}[\mathrm{~d} X] \operatorname{det}^{n_{A}-m}(X) e^{\operatorname{Tr}(\Lambda X)} \propto \int_{0}^{1} \cdots \int_{0}^{1} \Delta(x)^{2} \prod_{j=1}^{m} x_{j}^{n_{A}-m} \mathrm{~d} x_{j} \int \mathrm{d} \mu_{\text {Haar }}(U) e^{\operatorname{Tr}\left(\Lambda U \operatorname{diag}(x) U^{+}\right)}
$$

W.l.o.g, we assume that $\Lambda$ is in the diagonal form, i.e. $\Lambda=\operatorname{diag}\left(\lambda_{1}, \ldots, \lambda_{m}\right)$ where each $\lambda_{j} \in \mathbb{R}$. By Harish-Chandra-Itzykson-Zuber integral formula $[3,5]$,

$$
\int_{\mathrm{U}(m)} \mathrm{d} \mu_{\text {Haar }}(U) e^{\operatorname{Tr}\left(A U B U^{+}\right)}=\left(\prod_{j=1}^{m} \Gamma(j)\right) \frac{\operatorname{det}\left[\exp \left(a_{i} b_{j}\right)\right]}{\Delta(a) \Delta(b)},
$$

it follows that

$$
\int \mathrm{d} \mu_{\text {Haar }}(U) e^{\operatorname{Tr}\left(\Lambda U \operatorname{diag}(x) U^{+}\right)}=\left(\prod_{j=1}^{m} \Gamma(j)\right) \frac{\operatorname{det}\left[\exp \left(\lambda_{i} x_{j}\right)\right]}{\Delta(\lambda) \Delta(x)} .
$$

Therefore

$$
\begin{aligned}
\int_{0}^{\mathbb{1}_{m}}[\mathrm{dX}] \operatorname{det}^{n_{A}-m}(X) e^{\operatorname{Tr}(\Lambda X)} & \propto \frac{1}{\Delta(\lambda)} \int_{0}^{1} \cdots \int_{0}^{1} \Delta(x) \operatorname{det}\left[\exp \left(\lambda_{i} x_{j}\right)\right] \prod_{j=1}^{m} x_{j}^{n_{A}-m} \mathrm{~d} x_{j} \\
& \propto \frac{1}{\Delta(\lambda)} \int_{0}^{1} \cdots \int_{0}^{1}|\Delta(x)| \prod_{j=1}^{m} e^{\lambda_{j} x_{j}} x_{j}^{n_{A}-m} \mathrm{~d} x_{j},
\end{aligned}
$$

implying that

$$
\int_{0}^{\mathbb{1}_{m}}[\mathrm{~d} X] \operatorname{det}^{n_{A}-m}(X) e^{\operatorname{Tr}(\Lambda X)} \propto \frac{1}{\Delta(\lambda)}\left(\prod_{j=1}^{m} \partial_{\lambda_{j}}^{n_{A}-m}\right) \int_{0}^{1} \cdots \int_{0}^{1}|\Delta(x)| \prod_{j=1}^{m} e^{\lambda_{j} x_{j}} \mathrm{~d} x_{j} .
$$


Remark 4.5. Kumar in [7] have presented analytical formula for distribution of the sum of two Wishart matrices in terms of the confluent hypergeometric function of matrix argument [8], which is defined by

$$
{ }_{1} F_{1}(a ; c ;-\Lambda)=\frac{\Gamma_{p}(c)}{\Gamma_{p}(a) \Gamma_{p}(c-a)} \int_{0}^{\mathbb{1}_{p}} \operatorname{det}(X)^{a-p} \operatorname{det}(\mathbb{1}-X)^{c-a-p} e^{-\operatorname{Tr}(\Lambda X)}[\mathrm{d} X],
$$

where $\Gamma_{p}(a):=\pi^{\frac{p(p-1)}{2}} \Gamma(a) \Gamma(a-1) \cdots \Gamma(a-p+1)$. Kummer's formula for the confluent hypergeometric function ${ }_{1} F_{1}$ is given by

$$
{ }_{1} F_{1}(a ; c ;-\Lambda)=e^{-\operatorname{Tr}(\Lambda)} \cdot{ }_{1} F_{1}(c-a ; c ; \Lambda) .
$$

Remark 4.6. We can present a simple approach to the similar result. Denote $W_{m}^{\mathrm{C}}(n, \Sigma)$ Wishart matrix ensemble for which each matrix is of the form $W=A A^{*}$, where $A \in \mathbb{C}^{m \times n}(m \leqslant n)$. Now $W=W_{1}+W_{2}$, where $W_{1}, W_{2} \in W_{m}^{\mathrm{C}}(n, \Sigma)$, can be rewritten as

$$
W=Z Z^{\dagger}, Z=[A, B] \in \mathbb{C}^{m \times 2 n} .
$$

Then $W \sim W_{m}^{\mathrm{C}}(2 n, \Sigma)$. Thus

$$
\mathbf{P}_{W}(W) \propto \operatorname{det}^{2 n-m}(W) e^{-\operatorname{Tr}\left(\Sigma^{-1} W\right)}
$$

The distribution of sum of an arbitrary finite number of Wishart matrices can be derived as:

$$
\mathbf{P}_{W}(W) \propto \operatorname{det}^{k n-m}(W) e^{-\operatorname{Tr}\left(\Sigma^{-1} W\right)},
$$

where $W=\sum_{j=1}^{k} W_{j}$ for $W_{j} \sim W_{m}^{\mathbb{C}}(n, \Sigma)$.

\section{References}

[1] A. Lovas, A. Andai, Volume of the space of qubit-qubit channels and state transformations under random quantum channels, arXiv: 1708.07387

[2] M. Christandl, B. Doran, S. Kousidis, M. Walter, Eigenvalue distributions of reduced density matrices, Comm. Math. Phys. 332, 1-52 (2014).

[3] Harish-Chandra, Spherical Functions on a Semisimple Lie Group, I, Amer. J. Math. 80, 241-310 (1958).

[4] R.F. Hoskins, Delta Functions: An Introduction to Generalised Functions, 2nd Edition 2009, Woodhead Publishing Limited, Oxford (2011).

[5] C. Itzykson and J.B. Züber, The planar approximation. II, J. Math. Phys. 21, 411-421 (1980). 
[6] A.T. James, Distributions of Matrix Variates and Latent Roots Derived from Normal Samples, Ann. Math. Stat. 35(2), 475-501 (1964).

[7] S. Kumar, Eigenvalue statistics for the sum of two complex Wishart matrices, Europhys. Lett. 107, 60002 (2014).

[8] A.M. Mathai, Jacobians of Matrix Transformations and Functions of Matrix Arguments, World Scientific (1997).

[9] J. Mejía, C. Zapata, A. Botero, The difference between two random mixed quantum states: exact and asymptotic spectral analysis, J. Phys. A : Math. Theor. 50, 025301 (2017).

[10] S.R. Moghadasi, Polar decomposition of the $k$-fold product of Lebesgue measure on $\mathbb{R}^{n}$, Bull. Aust. Math. Soc. 85, 315-324 (2012).

[11] L. Zhang, Volumes of orthogonal groups and unitary groups, arXiv:1509.00537

[12] L. Zhang, Average coherence and its typicality for random mixed quantum states, J. Phys. A : Math. Theor. 50, 155303 (2017).

[13] K. Życzkowski, W. Słomczynski, Induced measures in the space of mixed quantum states, J. Phys. A : Math. Gen. 34, 7111 (2001). 